\title{
Berth Allocation Optimization with Priority based on Simulated Annealing Algorithm
}

\author{
Wang Yuping ${ }^{1}$, Hao Yangyang ${ }^{2, *}$, Zhang Yuanhui ${ }^{3}$, Huang Youfang ${ }^{2}$ and Gu Tianyi ${ }^{4}$ \\ ${ }^{1}$ Information Office, Shanghai Maritime University, Shanghai 201306, China \\ ${ }^{2}$ Logistics Research Center, Shanghai Maritime University, Shanghai 201306, China \\ ${ }^{3}$ Construction and Installation Branch Company, Laigang Construction Co. Ltd, Laiwu 271104, China \\ ${ }^{4}$ Department of Computer Science, University of New Hampshire, Durham, NH 03824, United States
}

Received 29 September 2017; Accepted 29 January 2018

\begin{abstract}
Existing berth-quay crane (QC)-integrated allocation models aim to minimize the total time of vessels at port or the total cost of vessels' berthing and handling. The service fairness of vessels is considered, but service priority to VIP customers is overlooked, thereby causing poor service satisfaction from berthing operations. A multi-objective continuous berth allocation model with priority was proposed in this study to address this problem. The proposed model explored the relationship among penalty cost, fairness, and priority of VIP customers and designed weight vectors for penalty cost and fairness on the basis of an analysis of berth waiting time, preference berth allocation, and QC allocation. A two-factor neighbourhood searching strategy was then implemented for berthing sequence and berthing position through the simulated annealing algorithm with considerations for priority, thereby solving the model rapidly. Different parameter strategies in the model were compared and verified by experiments. Results demonstrate that the continuous berth allocation model with priority ensures fairness of overall service in berth allocation and priori berthing of VIP customers. The simulated annealing algorithm can be superior to other heuristic algorithms in solving the model. Conclusions in this study offer theoretical bases for berthing service satisfaction on the port side and are conducive to the optimization of berth allocation.
\end{abstract}

Keywords: complex berth-QC, fairness, VIP customer service, neighbourhood searching, simulated annealing algorithm

\section{Introduction}

The Port of Shanghai, the largest container port in the world, suffered serious congestion in April 2017. The congestion lasted for a long time and expanded to surrounding ports continuously. Experts in the industry determined that this congestion was the consequence of shipping alliance adjustment, severely overloaded operation in the port, and system adjustment. As important nodes in water transportation, container terminals are important in operation efficiency. The berth-quay crane (QC) allocation of vessels after arrival at port is a primary task of ports. A reasonable berthing plan can reduce the berthing time of vessels effectively and thereby relieve congestion at the port. Berthing operation at port benefits multiple parties. Therefore, the formulation of high-efficiency and highservice-fairness berthing plans has become the key research content in the academe.

Current studies on berth-QC integrated allocation aim to minimize the total berthing time of vessels and service cost of ports and construct an optimization model that uses berthing time, berthing position, and allocated QC quantity as key factors, thus formulating an optimal scheduling scheme for vessels and resource allocation. Most continuous berth optimization models maximize the utilization of QC and berths by constructing berth and QC allocation models [1] or minimize the berthing cost of vessels in terms of fuel

\footnotetext{
*E-mail address: yyhoo@shmtu.edu.cn

ISSN: 1791-2377 @ 2018 Eastern Macedonia and Thrace Institute of Technology. All rights reserved. doi:10.25103/iestr.111.09
}

consumption and carbon emission [2]. These two methods emphasize the benefits of single parties and overlook the overall balance of benefits among different parties, general service fairness, and overall service satisfaction. A recent study constructed a continuous berth-QC allocation model that is based on fairness maximization [3]. This model emphasizes the benefit fairness between vessels and ports but overlooks the berthing priority of VIP customers in practical situations. Therefore, evaluating the benefit pursuit of vessels and ports realistically and comprehensively, improving service satisfaction, analyzing penalty costs and fairness of berthing operations, reflecting the appeal of overall service satisfaction of all parties, protecting the priority of VIP customers, and optimizing continuous berth arrangement are practically significant..

\section{State of the art}

Existing studies on continuous berth allocation optimization mainly concentrated on models and algorithms. Models were theories constructed with consideration for multiple factors, such as berth waiting time, berthing sequence, and quantity of QC allocation. Algorithms were processes of optimally solving the proposed models. With respect to modeling, all studies focused on optimizing the three key factors of berthing, namely, optimization of berthing time, optimization of berth allocation, and maximization of QC allocation. The optimization goals were generally set as minimization of berthing time and total operation cost of vessels and maximization of operation efficiency of ports. Fan [4] studied the continuous berth allocation of vessels 
under dynamic arrival conditions. A model that regards the minimization of total berthing time as the objective function was constructed and solved by the genetic algorithm. Zhu et al. [5] constructed a berth allocation model that treats the cost of deviation from the preference berth as the objective function and solved it by the genetic algorithm, thus reducing the extra cost of the deviation from the preference berth. Meisel et al. [6] expressed the handling time of vessels by the handling efficiency of QC and discussed the berth-QC integrated allocation problem. However, the authors did not consider the benefits for ship owners. Liang et al. [7] believed that the handling time of vessels is closely related to the handling efficiency of $\mathrm{QC}$ and discussed berth-QC combined allocation. Han et al. [8] studied a twostage model. In the first stage, a space-time continuous berth allocation model of vessels was constructed. In the second stage, the operation efficiency of the model was increased by reducing the movement of the bridge crane and minimizing the crane distribution interval. Han et al. [9] studied the QC scheduling problem from the uncertainty in the arrival and operation times of vessels, proposed a mix integer model, and solved the optimal scheduling by the genetic algorithm. The above studies have implied that minimizing the berthing time of vessels is another goal of optimization. Gui et al. [10] conducted an integral study of QC allocation and berthing schedule. The researchers constructed a continuous berth allocation model that is based on priority and solved it by a hybrid genetic algorithm to achieve the shortest total berthing and workloading times of vessels. He [11] proposed an integrated simulation-optimization method from the perspective of energy saving and emission reduction to reduce the penalty cost caused by delayed departure and decrease energy consumption cost. Qin et al. [12] discussed the berth allocation model from the perspective of water level changes. Ursavas [13] proposed a new bridge crane that can solve the problem of ordinary QC restriction by power supply and operation control cables. This new bridge crane could move freely on the port but showed poorer handling efficiency than ordinary QC did. Therefore, the bridge crane can only be a supplementation under special situations. Emde et al. [14] designed a portable mobile QC that can be placed on vehicles to serve as supplementation to ordinary QC. Nam and Lee [15] proposed a QC with floating platforms that can operate at two sides of vessels simultaneously, thus increasing handling efficiency. $\mathrm{Ku}$ and Arthanari [16] suggested synchronous loading and unloading. This operation mode unloads goods from the vessels to the port and then loads goods to vessels, thus avoiding nonoccupation operation. In summary, these studies have analyzed the penalty cost for delayed arrival, delayed departure, and deviation from the preference berth. However, the use of fairness in berth waiting, departure waiting, and QC quantity of vessels as the evaluation standards has rarely been explored.

With an increase in service level, the satisfaction and fairness of vessels and ports during service become important factors in scheduling optimization. Relevant studies have mainly used heuristic algorithms. Shan [17] constructed a dynamic continuous berth allocation model with priority by considering the effects of handling efficiency on berthing time and priority of vessels in actual operations. Golias et al. [18, 19] proposed grading berthing services for vessels according to priority agreements and solved the multi-objective optimization problem by the heuristic genetic algorithm. Sahin and Kuvvetli [20] considered berth allocation as a continuous spatial allocation problem, proposed a dynamic berth arrangement strategy, and solved their model by the differential evolution algorithm. Kim et al. [21] constructed a hybrid integer linear programming model to study the continuous berth allocation problem and solved it by the simulated annealing algorithm on LINDO. Liu et al. [22] focused on secondary berth allocation after accidental interruption of vessel operation to optimize berth allocation, reduce cost, and increase service satisfaction. Golias and Portal [23] studied a robust berth arrangement strategy that aims to reduce the total service time span and average service time of vessels in the port. the authors defined the problem of secondary optimization and proposed the use of a heuristic algorithm to solve this problem. However, the overall description was ambiguous. Lee et al. [24] defined port as a continuous space and optimized it through the neighborhood searching algorithm. First-come-first-service, waiting time, and dislocation of vessels were considered in service strategies. Lai and Shih [25] studied the berth utilization in the Hong Kong International Terminals and found that the organization proposed the concept of berthing priority to enhance competitiveness. Akio Imai [26,27] introduced the Lagrange relaxation algorithm to guarantee the shortest total berthing time of vessels and analyzed the importance of berthing priority in port operation with consideration for service priority factors. Fan et al. [28] discussed the fair allocation of QC resources and constructed a berth-QC scheduling model that considers the minimization of total berthing time as the objective function. This model was solved by the improved genetic algorithm and verified by a case study. Wang et al. [3] discussed continuous berth allocation that is based on fairness maximization and proposed a three-factor neighborhood searching algorithm. The optimal solution was searched rapidly by the simulated annealing algorithm. However, the researchers neglected the berthing priority of VIP customers.

Berthing service involves the benefits of multiple parties; therefore, optimizing berth allocation by considering the benefit of only one party is disadvantageous for improving service satisfaction. Most existing optimization models for berth arrangement do not consider the general benefit balance and service satisfaction of VIP customers. The contradictions of berth allocation still exist. Hence, a multiobjective continuous berth allocation model with priority was constructed by the present work to achieve berthing fairness. The proposed model analyzes berth waiting time, berth location, QC resource allocation, and influencing factors of berthing priority. The model solves the benefit balance among different parties and service satisfaction of VIP customers.

The remainder of this study is organized as follows. Section 3 describes the problems and hypotheses, constructs the studying model, designs the simulated annealing algorithm, and defines the neighborhood searching strategy. Section 4 explains the experimental design and result analysis. Results are compared with those of the neighborhood searching algorithm. Section 5 concludes the study.

\section{Methodology}

Dispatchers formulate berthing plans according to relevant information and distribute berth and QC to vessels. Shipping companies desire the shortest time of vessels in port and 
berthing at the preference berth. The port pursues the maximum container throughput and utilization of port resources. Therefore, berth-QC allocation optimization should distribute berth and QC resources reasonably according to arrival time, increase utilization, reduce the operation cost of the port, minimize berthing time, and improve customer service.

This study discussed the continuous berth arrangement problem. Vessels berth according to terminal markers. If the vessel length is less than the terminal length, then vessels are allowed to berth. Multiple vessels can be served simultaneously. In this study, fairness (that is, fairness of order, service time, and resource allocation and priority of VIP customers) of vessel service in the port was evaluated comprehensively according to expected arrival time (EAT), priority, and handling demands. A continuous berth allocation model was constructed.

This study cited the partial definition from the Study of Continuous Berth Allocation Algorithm based on Fairness Maximization of Wang and expanded the original model. Reasonable hypotheses were proposed.

(1) Each vessel must berth, and repeated berthing is not allowed.

(2) The arrival time of vessels is not earlier than the expected time. The starting time of vessel berthing must be later than the arrival time.

(3) Adequate QC resources are available to meet vessel berthing. Therefore, berthing time is only related to cargo carrying capacity.

(4) Many berthing units are divided continuously along the terminal. Many vessels can berth at the same time.

(5) To reduce distance between berths and rear storage yard, each vessel is assigned one optimal preference berth, and deviation from the preference berth will increase the operation cost.

(6) Each vessel has one priority attribute that represents the importance level of the VIP. The higher the attribute value, the higher the priority given to the berthing of the vessel.

Factors unrelated to this study are omitted.

\subsection{BA model}

\subsubsection{Symbol descriptions}

1) $s v=\{1,2, \cdots, s v s\}$ is the set of vessels waiting for berth, where $v \in s v$.

2) $s p=\{1,2, \ldots, s p s\}$ is the set of berth, where $p, \rho, \beta \in s p$.

3) $s t=\{1,2, \ldots, s t s\}$ is the set of time quantum in one service period, where $t, \tau, \gamma \in s t$.

4) $e_{v}$ is the EAT of vessel $v$.

5) $a_{v}$ is the total berthing time of vessel $v$, that is, the total container loading and unloading time of the vessel (per $\mathrm{QC} * \mathrm{~h})$.

6) $b_{v}$ is the length of vessel $v$.

7) $d_{v}$ is the expected departure time (EDT) of vessel $v$.

8) $s_{v}$ is the preference berth of vessel $v$.

9) $c_{v}^{1}$ is the penalty cost unit for the deviation from the preference berth of vessel $v$.

10) $c_{v}^{2}$ is the penalty cost unit for the delayed berthing of vessel $v$.

11) $c_{v}^{3}$ is the penalty cost unit for the delayed departure of vessel $v$.
12) $l_{v}$ is the quantity of QC allocated to vessel $v$.

13) $M$ is a sufficiently large positive integer.

14) $w_{v}$ is the priority parameter of vessel $v$; the higher this value, the higher the priority.

15) $X_{v p t}$ is the operation sign of vessel $v$ in position $(p, t)$ in the space-time matrix. A value of 1 means that the vessel operates at this position.

16) $Z_{v p t}$ is 1 if point $(p, t)$ is the reference point; otherwise, it is 0 . Reference point refers to the left bottom of the rectangle, that is, the leftmost position at the initial berthing time and berthing position.

17) $V_{v, t}$ is 1 if vessel $v$ is operating at $t$; otherwise, it is 0 .

18) $U_{v, t}$ is 1 if vessel $v$ berths at $p$; otherwise, it is 0 .

19) $C_{v}$ is the spanning time of cargo loading and unloading.

20) $B L_{v}$ is the leftward deviation length of vessel $v$ from the preference berth.

21) $B R_{v}$ is the rightward deviation length of vessel $v$ from the preference berth.

22) $T L_{v}$ is the delay between the actual arrival of vessel $v$ and the EAT.

23) $D L_{v}$ is the delay between the actual departure of vessel $v$ and the EDT.

24) $B_{v}=\sum_{p, t}\left(Z_{v p t} \cdot p\right)$ is the occupied berth of vessel $v$.

25) $T_{v}=\sum_{p, t}\left(Z_{v p t} \cdot t\right)$ is the occupation time of vessel $v$.

26) $P O S_{p, t}=\sum_{v}\left(Z_{v p t} \cdot v\right)$ means that point $(p, t)$ is occupied by vessel $v$.

\subsubsection{Objective functions and model}

The objective function of the continuous berth-QC allocation model with priority is:

$g=w_{\theta} \cdot \frac{\theta^{\max }-\theta}{\theta^{\max }-\theta^{\min }}+w_{f} \cdot \frac{f-f^{\min }}{f^{\max }-f^{\min }}$

where $f$ is the sum of functions $f^{1}, f^{2}$, and $f^{3}$ in the model. $f^{\max }$ and $f^{\min }$ are the maximum and minimum values of the function. $\theta^{\max }$ and $\theta^{\text {min }}$ are the maximum and minimum values of the fairness function. Penalty cost and fairness measures synthesize one independent objective function $(g)$ through Equation (1). Under this circumstance, $w_{\theta}+w_{f}=1, w_{\theta} \geq 0$, and $w_{f} \geq 0$.

\section{(1) Penalty cost $(f)$}

The optimization goal of berth-QC allocation covered two aspects. One was the optimization of service time. Vessels should berth as soon as possible after arrival, accomplish loading and unloading in the regulated time of shipping companies after berthing, and then depart on schedule. The other aspect was the optimization of berths. The berth should be as close as possible to the preference to reduce the movement time of containers from QC to the rear storage yard. These two aspects had to consider the priority given to VIP customers. These two goals established an objective function of minimizing total penalty cost, which involved the priority. This function aimed to increase the satisfaction of VIP customers, reduce the penalty cost of berthing, and 
shorten the berth waiting time and delayed departure time. The objective function is defined by Equations (2)-(5), and relevant constraints are defined by Equations (6)-(24).

Minimize:

$$
\begin{aligned}
& f=\left(f^{1}, f^{2}, f^{3}\right) \\
& f^{1}=\sum_{v}\left(B L_{v}+B R_{v}\right) c_{v}^{1} \\
& f^{2}=\sum_{v} T L_{v} \cdot c_{v}^{2} \cdot w_{v} \\
& f^{3}=\sum_{v} D L_{v} \cdot c_{v}^{3} \cdot w_{v}
\end{aligned}
$$

Constraints:

$$
\begin{aligned}
& B L_{v} \geq B_{v}-S_{v}, \forall v \\
& B R_{v} \geq S_{v}-B_{v}, \forall v \\
& T L_{v} \geq T_{v}-e_{v}, \forall v \\
& T_{v} \geq e_{v}, \forall v \\
& D L_{v} \geq C_{v}-d_{v}, \forall v \\
& C_{v} \geq V_{s t} \cdot(t+1), \forall v \\
& \sum_{v} X_{v p t} \leq 1, \forall p, t \\
& V_{v t} \leq \sum_{p} X_{v p t}, \forall v, t \\
& V_{v t} \cdot M \geq \sum_{p} X_{v p t}, \forall v, t \\
& U_{v p} \leq \sum_{t} X_{v p t}, \forall v, p \\
& U_{v p} \cdot M \geq \sum_{p} X_{v p t}, \forall v, p \\
& (\beta-\rho+1) \leq \sum_{p, \sigma \geq p \geq o} U_{v p}+M\left(2-U_{v o}-U_{v \sigma}\right), \forall v, \rho<\beta \\
& (\gamma-\tau+1) \leq \sum_{p, \gamma \geq t \geq \tau} V_{v t}+M\left(2-U_{v \gamma}-U_{v \tau}\right), \forall v, \tau<\gamma \\
& V_{v t} \leq \sum_{p, \tau \leq t} Z_{v p \tau}, \forall v, t \\
& \sum_{p, t} Z_{v p t}=1, \forall v \\
& \sum_{p<\rho, t} X_{v p t}+\sum_{p \geq \rho+b_{v}, t} X_{v p t} \leq M\left(1-\sum_{t} Z_{v p t}\right) \\
& \forall v, \rho \geq 2, \rho \leq s p s-b_{v} \\
& \sum_{p>b_{v}, t} X_{v p t} \leq M\left(1-\sum_{t} Z_{v, 1, t}\right), \forall v \\
& \sum_{p>s m s-b_{v}, t} X_{v p t} \leq M\left(1-\sum_{t} Z_{\left.v, s m s-b_{k}+1, t\right), \forall v}\right. \\
& b_{k}-\sum_{p} X_{v p t} \leq M\left(1-V_{v t}\right), \forall v, t
\end{aligned}
$$

The penalty cost function is shown as Equation (2). The smaller the penalty cost, the better the fairness. This function covered the total cost for the deviation from the preference berth (Equation (3)), the total penalty cost for delayed berthing (Equation (4)), and the total penalty cost for delayed departure (Equation (5)). Equations (7) and (8) restrict the deviation distances from the preference berth. Equation (8) defines the delayed berthing time of the vessel. Equation (9) imposes the restriction that vessels can only berth after arrival at the port. Equation (10) shows the delayed departure time. Equation (11) determines that vessels can depart only after loading and unloading. Equation (12) restricts that each time-space point can be occupied by only one vessel. Equations (13) and (14) establish the relationship between $V_{k, j}$ and $X_{k i j}$. Equations (15) and (16) establish the relationship between $U_{k, i}$ and $X_{k i j}$. Equations (17), (18), and (19) ensure the time-space continuity of berth occupation by vessels. Equation (20) determines that one vessel can occupy only one time-space point at a time. Equations (21),(22),(23), and (24) determine that grids in the rectangle are valued 1 and non-occupied grids out of the rectangle are valued 0 .

(2) Fairness measures $(\theta)$

The priority of vessel berthing is expressed by $w_{v}$. The default value of $w_{v}$ is 1 . The higher the value, the higher the priority. The priority was linearly related to delayed berthing and departure times, which are against service fairness.

Vessels are ordered according to EAT as follows: $e_{1} \leq e_{2} \leq \ldots \leq e_{s v s}$. The service time of vessel $v$ is calculated by $\Psi_{v}=C_{v}-T_{v}, v \in s v$. By arrival time, the basic fairness of berthing order is as follows:

$$
\theta(\pi)=\frac{\sum_{n<m}\left(I\left(\pi_{n}^{a}\right) \leq I\left(\pi_{m}^{a}\right)\right)^{\wedge}\left(e_{\pi_{n}} \leq e_{\pi_{m}}\right)}{(s v s+1) s v s / 2}
$$

The order of vessels that are waiting for berths in the port service system was defined as $\pi \cdot \pi^{a}$ and $\pi^{d}$ were generated according to EAT and EDT. The element orders of $\pi^{a}$ and $\pi^{d}$ are $\pi^{a}=\left\langle\pi_{1}^{a}, \pi_{2}^{a}, \ldots, \pi_{s v s}^{a}\right\rangle$ and $\pi^{d}=\left\langle\pi_{1}^{d}, \pi_{2}^{d}, \ldots, \pi_{s v s}^{d}\right\rangle$. On this basis, the fairnesses of delay against EAT and EDT were explored.

Delay from EAT:

$\theta_{d a}(\pi)=\frac{\sum_{n+1=m}\left(\left(I\left(\pi_{n}^{a}\right) \leq I\left(\pi_{m}^{a}\right)\right) \wedge\left(a_{n} \leq a_{m}\right)\right) \bullet w_{v}}{s v s-1}$

Delay from EDT:

$\theta_{d d}(\pi)=\frac{\sum_{n+1=m}\left(\left(I\left(\pi_{n}^{d}\right) \leq I\left(\pi_{m}^{d}\right)\right) \wedge\left(a_{n} \leq a_{m}\right)\right) \bullet w_{v}}{s v s-1}$

Fairness of berth arrangement was discussed according to whether the berth was a preference one.

Non-preference berth: 
$\theta_{r d}(\pi)=\frac{\sum_{n<m}\left(\left(D L\left(\pi_{n}^{a}\right) \leq D L\left(\pi_{m}^{a}\right)\right) \wedge\left(a_{n} \leq a_{m}\right)\right)}{(s v s+1) s v s / 2}$

Preference berth:

$$
\theta_{r \psi}(\pi)=\frac{\sum_{n<m}\left(\left(\psi\left(\pi_{n}^{a}\right) \leq \psi\left(\pi_{m}^{a}\right)\right) \wedge\left(a_{n} \leq a_{m}\right)\right)}{(s v s+1) s v s / 2}
$$

The overall fairness function is the sum of the above fairness functions.

$$
\theta(\pi)=\sum_{*}\left(w_{*} \cdot \theta_{*}(\pi)\right)
$$

\subsection{Simulated annealing algorithm}

The simulated annealing algorithm was discussed in the Study of Continuous Berth Arrangement based on Fairness Maximization. This heuristic algorithm simulates the physical annealing process, which is composed of the heating, isothermal, and cooling processes. This algorithm is extremely effective in obtaining the optimal solution to continuous berth-QC allocation. The simulated annealing algorithm is different from other algorithms because it accepts new states with a certain probability, thus avoiding local optima.

In the simulated annealing algorithm, a new solution is mainly generated by the neighborhood searching algorithm. First, the neighborhood searching algorithm expands the searching range by changing the neighboring domain structure set systematically and finds the locally optimal solution. Second, the searching range is expanded again by changing the neighboring domain structure set on the basis of the locally optimal solution, and another locally optimal solution is determined. In this study, the neighborhood searching algorithm was divided into two stages. The first stage involved arranging the berthing time, and the second stage involved finding the best berth position.

\subsubsection{Strategy of neighboring domain generation}

In this study, the neighborhood searching algorithm required several parameters.

(1) Set of vessels ( $s v)$, set of berths ( $s p)$, and set of available berthing times ( $s t$ )

(2) Relevant information of vessel $v$ : $\operatorname{EAT}\left(e_{v}\right)$, working hours of QC $\left(a_{v}\right)$, vessel length $\left(b_{v}\right)$, EDT $\left(d_{v}\right)$, deviation from the preference berth $\left(s_{v}\right)$, penalty cost unit for the deviation from the preference berth $\left(c_{v}^{1}\right)$, penalty cost unit for delay from EAT $\left(c_{v}^{2}\right)$, penalty cost unit for delay from $\operatorname{EDT}\left(c_{v}^{3}\right)$, and priority of vessel $\left(w_{v}\right)$

These parameters were integrated into the berth allocation function. The berthing time $\left(T_{v}\right)$ and berth positions $\left(B_{v}\right)$ of all vessels were generated after $m_{p}$ iterations.

Two neighborhood domains were generated using the verified interchange sequence. The indices of two vessels in the interchange sequence $(\pi)$ are $i_{1}$ and $i_{2}$. Under this circumstance, the distribution of $i_{2}$ is $i_{2} \sim N\left(i_{1}, b^{2}\right)$, where $b=\max \left\{\left(i_{1}, s v s-i_{1}\right)\right\}$.
The normal distribution $N\left(A, B^{2}\right)$ can be generated by two uniform distributions $a \sim U(0,1)$ and $b \sim U(0,1)$. $x=A+B(\sqrt{-2 \ln a}) \sin (2 \pi b)$ when $x \sim N\left(A, B^{2}\right)$.

$i_{1}$ is calculated by random positioning. However, the random positioning has to be verified again when $w_{v}$ is involved. First, two adjacent vessels in the order $(\pi)$ are defined as $v$ and $v_{n}$, whose values are $r B_{v}$ and $r B_{v_{n}}$ under the corresponding value of $r B$. According to the relative positioning strategy, the position of vessel $v$ is expressed by $B_{v}$ and the position of $v_{n}\left(B_{v_{n}}\right)$ is expressed by $B_{v_{n}} \leftarrow\left(B_{n}+b_{v}+r B_{v_{n}}-B L_{v}+B R_{v}\right)$.

The acceptable adjustment probability of berth position is expressed by $m_{p}$, where $0 \leq m_{p} \leq 1$.

A random value was generated in the range of $[0,1]$ whenever the vessel moved to the preference berth. If this random value was smaller than $m_{p}$, then the adjustment was accepted.

\subsubsection{Cooling scheduling and initial temperature $T_{0}$}

Cooling scheduling is the cooling management table from one high-temperature state $\left(T_{0}\right)$ to the low-temperature state in the simulated annealing algorithm. In this study, the classical cooling mode was selected. The temperature at $t$ was supposed to be $T(t), T(t)=\frac{T_{0}}{\ln (1+t)}$. This classical cooling mode makes the simulated annealing algorithm converge at the globally minimum point.

The experiment demonstrated that the higher the initial temperature, the higher the probability of gaining a highquality solution. However, computation consumed considerable time. Therefore, the initial temperature should be determined by compromises between optimization quality and efficiency. Common approaches include uniform sampling and random sampling. Here, 50 groups of states were generated randomly, and the maximum difference of target values between two states $\left(\left|\Delta_{\max }\right|\right)$ was determined. The initial temperature was determined according to $\left|\Delta_{\max }\right|$ on the basis of function $T_{0}=\frac{-\Delta_{\max }}{p_{r}}$, where $p_{r}$ is the initial probability of acceptance.

\section{Result analysis and discussion}

\subsection{Experimental design}

This study applied the random data in Table 1 as the experimental data to verify the accuracy of the model and the validity of the proposed algorithm. In Table 1,18 vessels are waiting for berthing, and 600 length units of available berths exist. A contrast analysis on objective function values under different strategies and parameters was conducted.

(1) Objective function: The smaller the objective function, the more optimal the berth allocation and the better the fairness of the overall benefit distribution.

(2) Delayed berthing time of vessel with priority: The priority strategy became increasingly effective as 0 was approached. Vessels could berth on schedule. 
(3) Iteration times at minimum objective function: The fewer the iterations, the higher the reasonability of the parameter and the higher the solving efficiency of the algorithm.

Experimental contents of this algorithm mainly include the following.

(1) The minimum objective function value was estimated and the iteration times were recorded to evaluate the overall fairness of the model and solving efficiency of the algorithm.

(2) The objective function values and delayed berthing time of a vessel with and without priority were recorded to analyze the effects of priority on the berthing of the vessels.

(3) $m_{d}$ was determined in the range of $[1,100]$ at an adjustment interval of 5 . A reasonable value range of $m_{d}$ was observed.

Table 1. Data of original berthing vessels

\begin{tabular}{|c|c|c|c|c|c|c|c|}
\hline Vessel & Length & Arrival Time & Cranes & Workload(QC*h) & Preferred Berth Position & EDT & Priority \\
\hline 1 & 347 & 13 & 9 & 4 & 226 & 24 & 1 \\
\hline 2 & 382 & 13 & 10 & 21 & 15 & 23 & 1 \\
\hline 3 & 169 & 13 & 4 & 6 & 350 & 27 & 1 \\
\hline 4 & 219 & 8 & 5 & 20 & 37 & 21 & 1 \\
\hline 5 & 175 & 39 & 4 & 3 & 238 & 51 & 1 \\
\hline 6 & 64 & 13 & 2 & 5 & 212 & 23 & 1 \\
\hline 7 & 216 & 15 & 5 & 20 & 137 & 28 & 1 \\
\hline 8 & 199 & 19 & 5 & 20 & 333 & 30 & 99 \\
\hline 9 & 344 & 12 & 9 & 12 & 136 & 25 & 1 \\
\hline 10 & 344 & 18 & 9 & 6 & 29 & 33 & 1 \\
\hline 11 & 259 & 5 & 6 & 20 & 183 & 15 & 1 \\
\hline 12 & 248 & 34 & 6 & 16 & 145 & 44 & 1 \\
\hline 13 & 327 & 21 & 8 & 18 & 273 & 32 & 1 \\
\hline 14 & 92 & 40 & 2 & 5 & 219 & 50 & 1 \\
\hline 15 & 186 & 13 & 5 & 8 & 153 & 23 & 1 \\
\hline 16 & 318 & 36 & 8 & 12 & 249 & 48 & 1 \\
\hline 17 & 161 & 13 & 4 & 9 & 308 & 25 & 1 \\
\hline 18 & 301 & 33 & 8 & 24 & 142 & 47 & 1 \\
\hline
\end{tabular}

\subsection{Experimental results and analysis}

The results of continuous berth arrangement based on data in Table 1 are shown in Fig.1. Numbers in the vessel rectangle reflect the serial number of vessels and the waiting time. The upper left corner shows the berth position and berthing time of vessels. Width refers to the length of vessels, and height is the operation time of the vessels after berthing. The blue border reflects zero berth waiting time, and the red framework reflects certain berth waiting times. The
(4) The probability of berth position acceptance was adjusted in the range of $[0,1]$ to evaluate its impacts on the solving efficiency of the algorithm.

(5) The efficiencies of the neighborhood searching algorithm and the simulated annealing algorithm with priority of vessels were compared. The condition for terminating iterations in the neighborhood searching algorithm was set as 3,000 iterations. The condition for terminating iterations in the simulated annealing algorithm was set as 1,000 iterations or over 30 rejections of the new solution. The solving qualities and efficiencies of the two algorithms were compared.

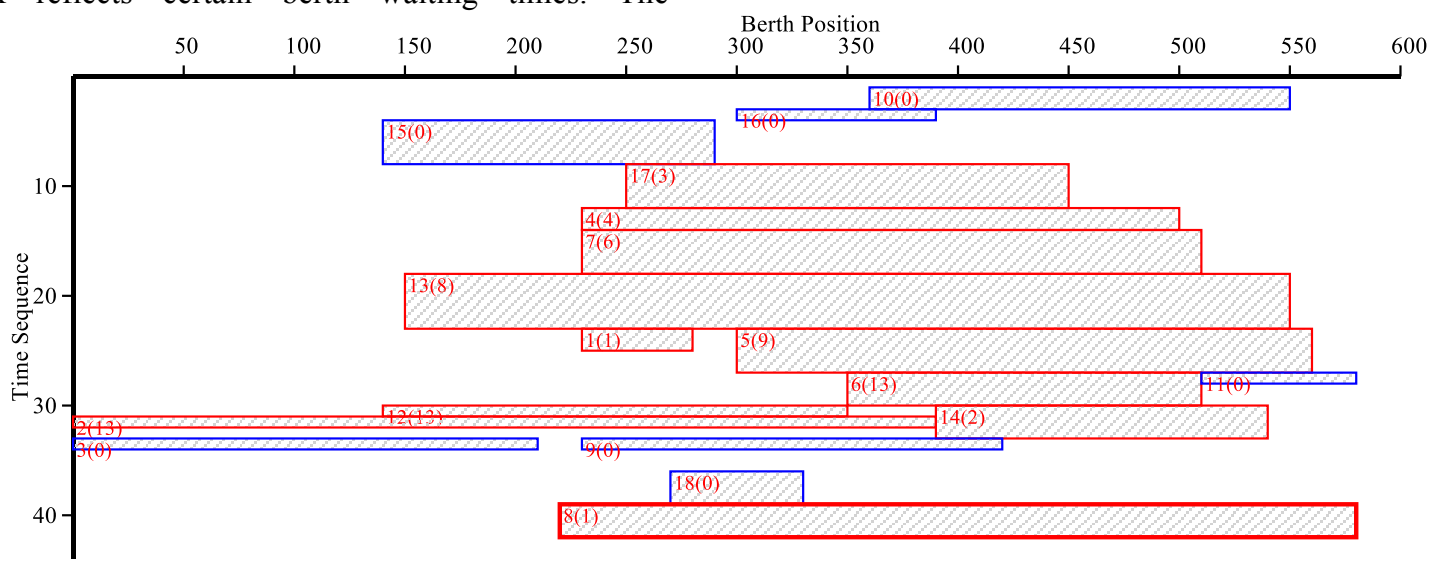

(a) Random solution

rectangle with thick borders shows certain priorities of the vessels. Fig.1(a) depicts a random solution. Fig.1(b) illustrates the optimal solution of the neighborhood searching algorithm. Finally, Fig.1(c) shows the optimal solution of the proposed algorithm and demonstrates that this solution optimized the berth position and waiting time of the vessel. 
Wang Yuping, Hao Yangyang, Zhang Yuanhui, Huang Youfang and Gu Tianyi/

Journal of Engineering Science and Technology Review 11 (1) (2018) 74-83

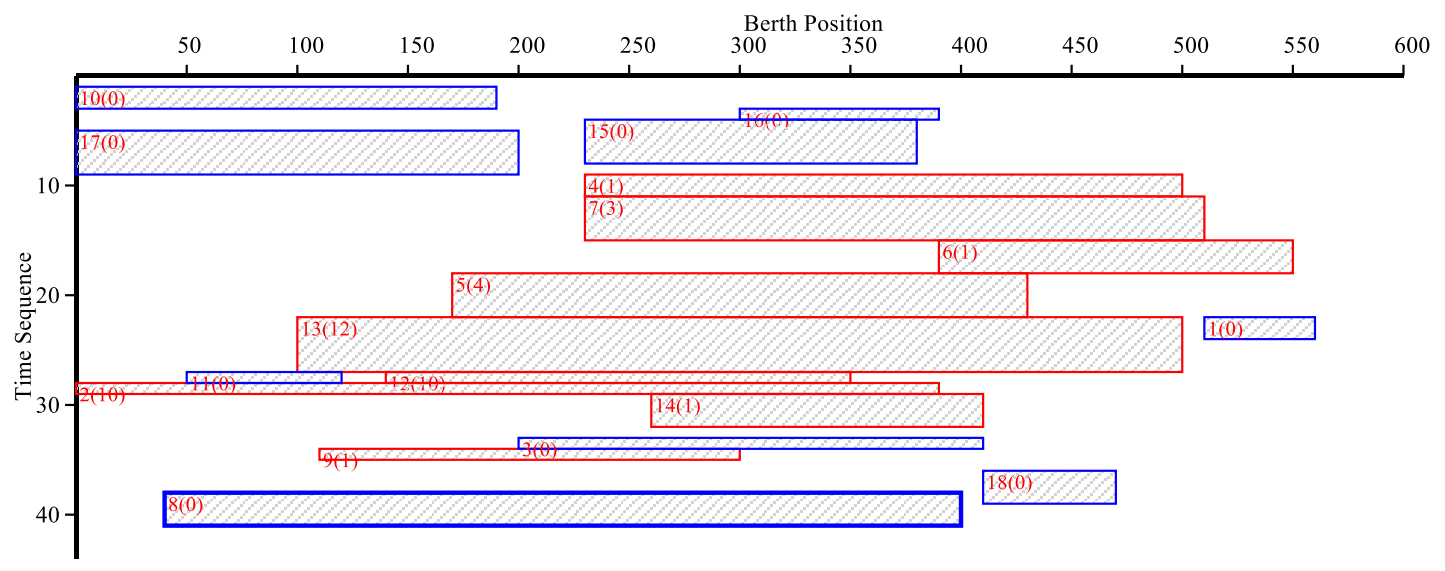

(b) Solution of neighborhood searching algorithm

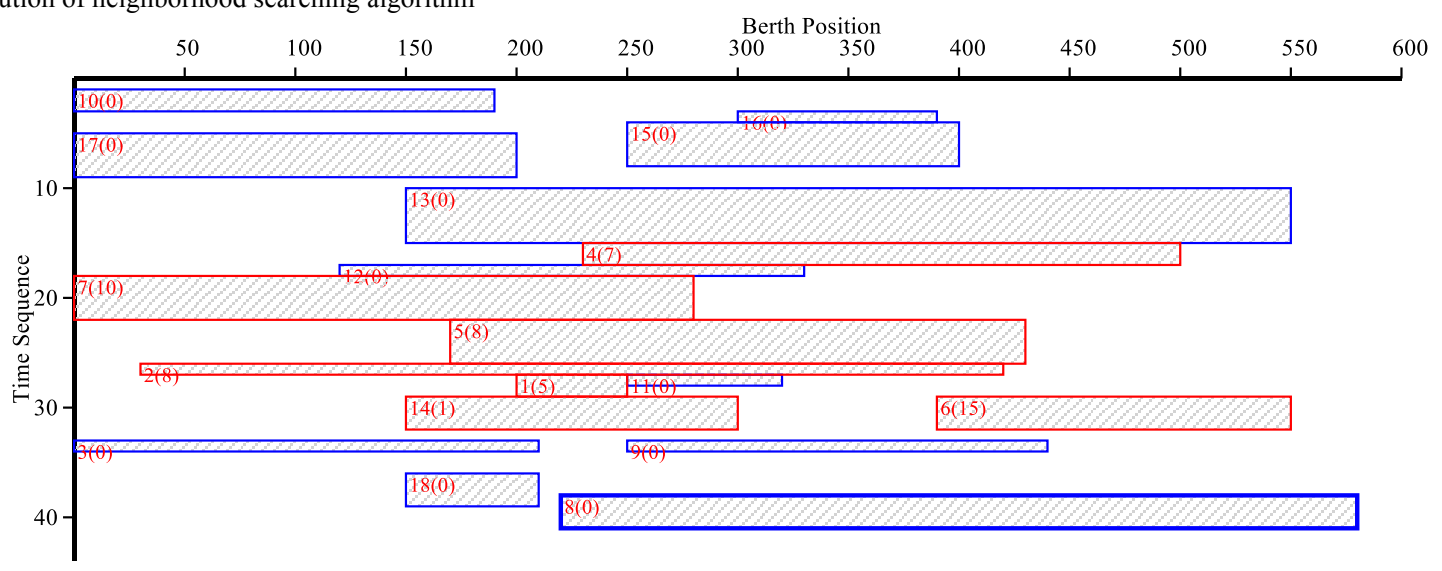

(c) Solution of simulated annealing algorithm

Fig. 1 Comparison of continuous berth allocation among different algorithms

The berth allocation results of the $8 \#$ vessel in the simulated annealing algorithm with and without priority are shown in Fig.2. The berth allocation of the 8\# vessel in the simulated annealing algorithm without priority is shown in Fig.2(a), in which the $8 \#$ vessel has to wait for 9 unit time before berthing. The berth arrangement of the $8 \#$ vessel in the simulated annealing algorithm with 99 priority is shown in Fig.2(b), in which the 8\# vessel can berth immediately upon arrival. This comparison shows that important customers could be served first in a long queue by increasing their priority.



(a) No priority to $8 \#$ vessel 
Wang Yuping, Hao Yangyang, Zhang Yuanhui, Huang Youfang and Gu Tianyi/

Journal of Engineering Science and Technology Review 11 (1) (2018) 74-83

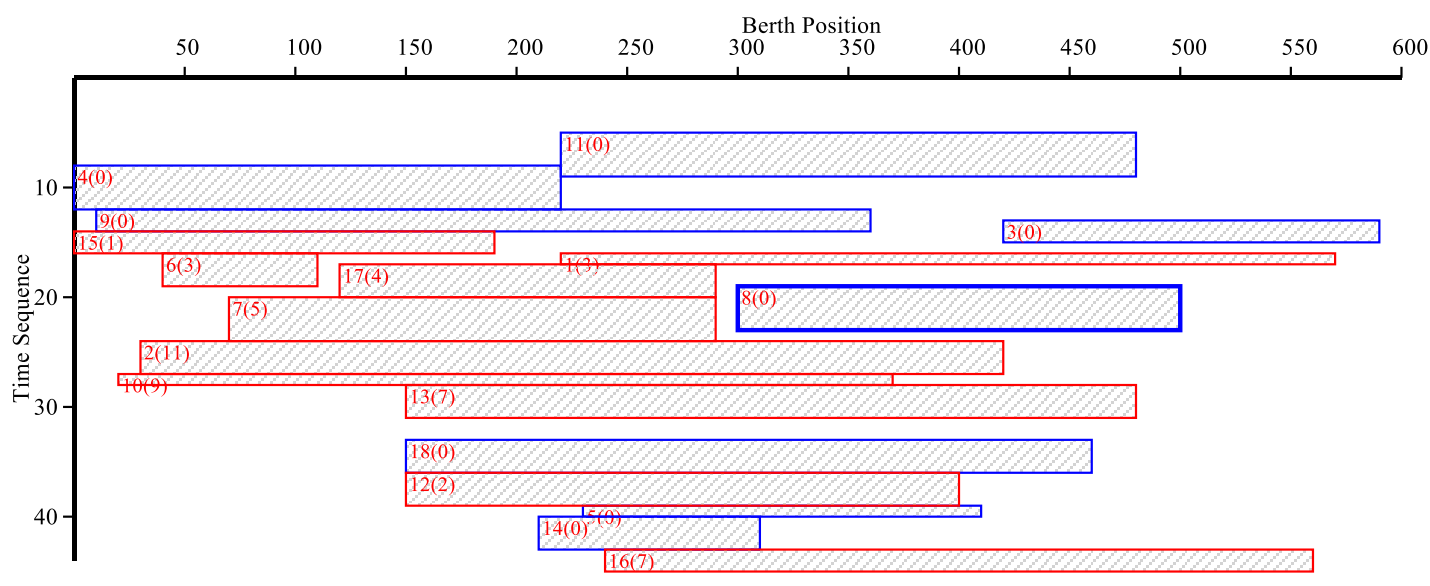

(b) Priority to $8 \#$ vessel

Fig. 2 Comparison of optimal scheduling programs with and without priority

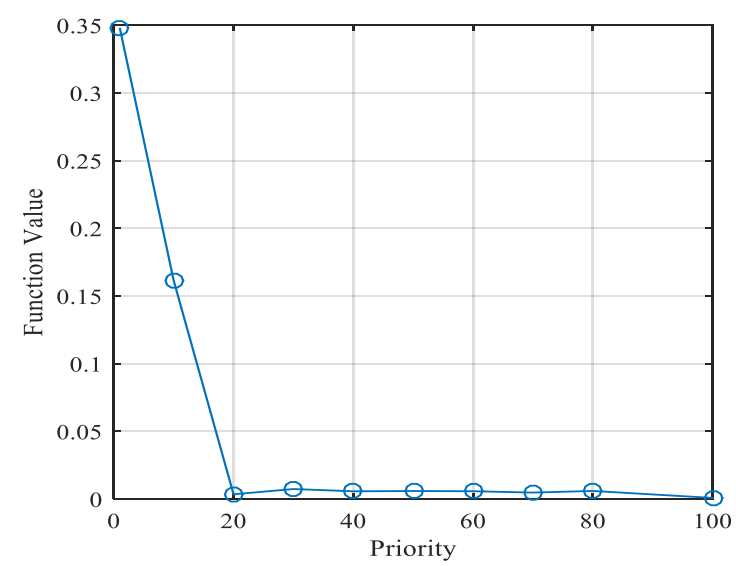

(a) Objective function values under different degrees of priority

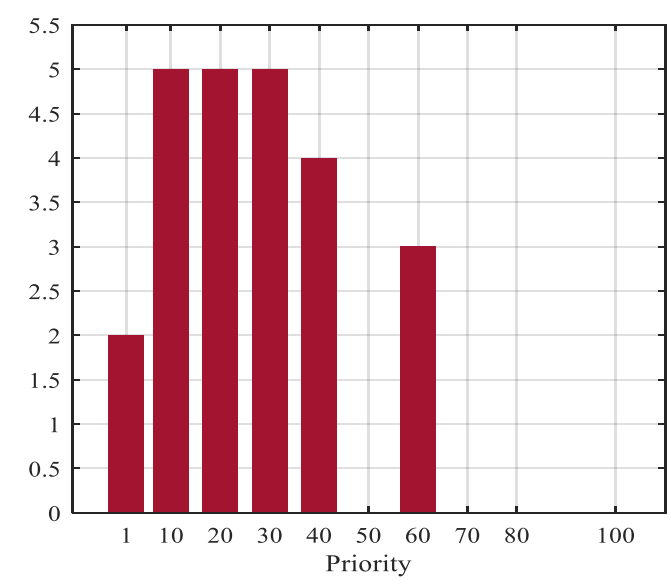

(b) Delay time under different levels of priority

Fig. 3 Influence of priority on berth allocation

The berth allocation under different levels of priority is shown in Fig.3. Fig. 3(a) depicts that a high priority was conducive to increasing the probability of prompt service. In Fig.3(b), vessels with priority $>70$ can berth immediately upon arrival. The independence of the optimal objective function value from priority was thus validated. Priority was a given condition and the premise to obtain the optimal solution. The objective function values under different levels of priority were incomparable. In practical port operation, only VIP customers with $>70$ priority can be served immediately after arrival.
The process of the simulated annealing algorithm is shown in Fig.4. The solution sequence was generated according to iteration times, which recorded the acceptable minimum, thus forming the sequence of acceptable values. The minimum of this sequence was the solution of the simulated annealing algorithm. In this experiment, the optimal solution (0.0025) was acquired at nearly 50 iterations. On this basis, the overall fairness was determined.

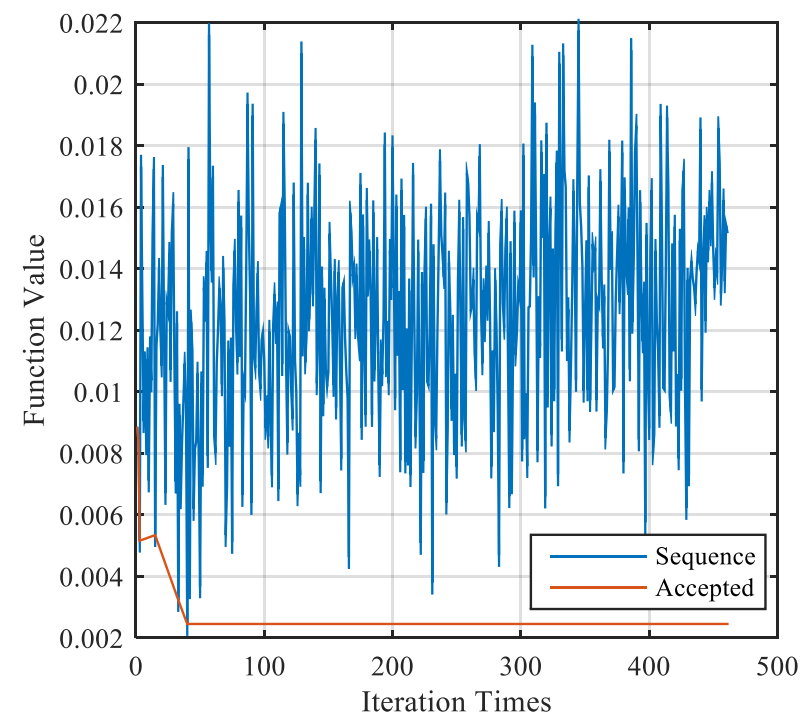

Fig. 4 Iteration process of simulated annealing algorithm

In the solving process of the simulated annealing algorithm, $m_{d}$ refers to the iteration times in the solving process. $m_{d}$ is a key parameter that influences the solving efficiency of the algorithm. Fig.5 indicates that with an increase in $m_{d}$ in the range of $[1,100]$, the overall reduction of the objective function value was insignificant. However, the objective function value with priority fluctuated considerably. Therefore, the reasonable value range of $m_{d}$ was determined to be within $[20,100]$ with reference to the original algorithm. Moreover, the range of $m_{d}$ was insensitive to priority. In actual calculation, any value in the range of $[20,100]$ is acceptable and will not affect the solution. 


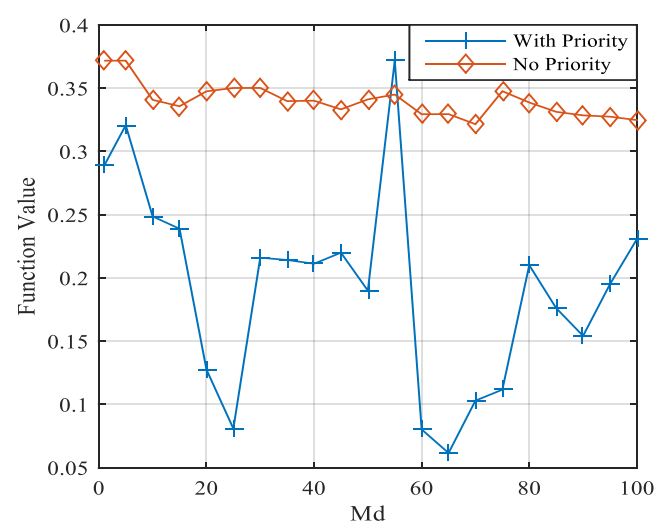

Fig. 5 Effect of priority on $m_{d}$

Solutions from the neighborhood searching and simulated annealing algorithms are shown in Fig.6. Significant differences were observed between these two algorithms. The optimal solution of the neighborhood searching algorithm was kept at 0.005 after 1,000 iterations. Meanwhile, the solution of the simulated annealing algorithm reached 0.004 after 190 iterations. The simulated annealing algorithm could produce the optimal berth allocation faster. The berth allocation based on the simulated annealing algorithm achieved better fairness and saved 5/6 of time than that based on the neighborhood searching algorithm did.

\section{Conclusions}

Reducing the time of vessels stay in port and increasing port operation efficiency can only provide benefit for single party but for every party. To improve the service satisfaction of VIP customers, and balance the benefits of every party, this study established a multi-objective continuous berth-QC itegrated allocation model with priority and based on service fairness. The model involved weight coefficients of priorities and fulfilled the priori berth allocation of VIP customers. The following conclusions could be drawn:
(1) VIP customers can gain berth allocation and preference berth as much as possible by increasing the priority parameter, thus enhancing the service satisfaction of these customers.

(2) The simulated annealing algorithm is applicable to the fast solving of the berth allocation model with priority. This algorithm can achieve the optimal solution after only a few iterations and has higher efficiency than other heuristic algorithms have.

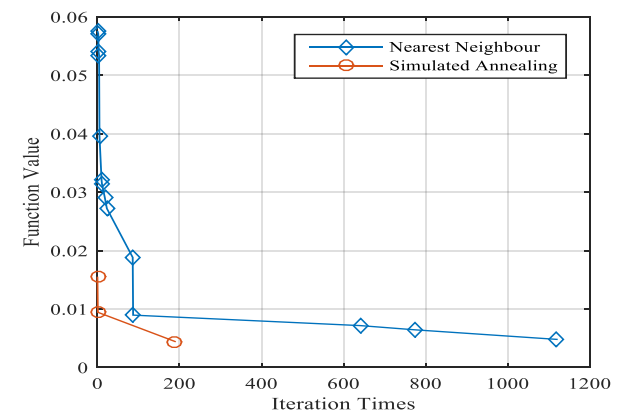

Fig. 6 Comparison between neighborhood searching and simulated annealing algorithms

The constructed multi-objective continuous berth-QC allocation model with priority ensures the service priority of VIP customers. However, theoretical support for the setting of priority weights is lacking. Future studies should discuss the relationship between priority weight and number of vessel sequences and the influence of this relationship on overall service fairness in berth allocation to further increase the accuracy and matching degree of the model in practical situations.

This is an Open Access article distributed under the terms of the Creative Commons Attribution Licence

\section{References}

1. Zhao K., Han X., Liang C., "Berth and Quay Crane Collaborative Optimization Research at Continuous Terminals". Journal of Wuhan University of Technology, 33(11), 2011, pp.60-65.

2. Hua Q., Hu Z., Du Y., "Berth and quay-crane allocation problem considering fuel consumption and emissions from vessels". Computers \& Inductrial Engineering, 70, 2014, pp.1-10.

3. Wang Y., Xiao Z., Huang Y., et al. "Study of Continuous Berth Allocation Algorithm Based on Fairness Maximization". Journal of Engineering Science and Technology Review, 10(5), 2017, pp.116127.

4. Fan Z., "Research on Continuous Berth Allocation Problem:Model Comparison and Computational Analysis". Industrial Engineering and Management, 3(21), 2016, pp.81-87.

5. Zhu S., Jin Y., "Berth-Crane Allocation Problem Based on MultiAgent with the Preferences of Berthing Position". Modern Computer, 20, 2016, pp.39-44.

6. Frank M., Christian B., "Integration of berth allocation and crane assignment to improve the resource utilization at a seaport container terminal'. Berlin: Springer, Germany, 2005, pp.105-110.

7. Liang, C., Huang, Y., Yang Y., "A quay crane dynamic scheduling problem by hybrid evolutionary algorithm for berth allocation planning". Computers \& Industrial Engineering, 56(3), 2009, pp.1021-1028.
8. Han, X. L., Gong, X., and Jo J., "A new continuous berth allocation and quay crane assignment model in container terminal". Computers \& Industrial Engineering, 89, 2015, pp.15-22.

9. Han, X. L., Lu, Z. Q., Xi, L. F., "A proactive approach for simultaneous berth and quay crane scheduling problem with stochastic arrival and handling time". European Journal of Operational Research, 207(3) 2010, pp. 1327-1340.

10. Gui X., Lu Z., Han X., Integrating Optimization Method for Continuous Berth and Quay Crane Scheduling in Container Terminals. Journal of Shanghai Jiaotong University, 47(02), 2013, pp. 226-229.

11. He, J., "Berth allocation and quay crane assignment in a container terminal for the trade-off between time-saving and energy-saving". Advanced Engineering Informatics, 30, 2016, pp.390-405.

12. Qin, T., Du, Y., Sha, M., "Evaluating the solution performance of IP and CP for berth allocation with time-varying water depth". Transportation Research Part E, 87, 2016, pp.167-185.

13. Ursavas E., "A decision support system for quayside operations in a container terminal". Decision Support Systems, 59, 2014, pp.312-324.

14. Emde S., Boysen N., Briskorn D., "The berth allocation problem with mobile quay walls: problem definition, solution procedures, and extensions". Journal of Scheduling, 17(3), 2014, pp. 289-303. 
15. Nam H., Lee T., "A scheduling problem for a novel container transport system: a case of mobile harbor operation schedule". Flexible Services and Manufacturing Journal. 25(4), 2013, pp.576608 ..

16. Ku D., Arthanari, T. S., "On double cycling for container port productivity improvement". Annals of Operations Research, 243(1), 2016, pp.55-70.

17. Tong, S., "Solving Dynamic Berth Allocation Problem at Container Terminal Based on Ships' Service Priority”. Journal of Transportation Engineering and Information, 10(1), 2012, pp.105110 .

18. Golias, M. M., Boile, M., Theofanis, S., "A lamda-optimal based heuristic for the berth scheduling problem". Transportation Research Part C: Emerging Technologies, 18(5), 2010, pp.794-806.

19. Golias, M. M., Boile, M., Theofanis, S., “. Berth scheduling by customer service differentiation: A multi-objective approach". Transportation Research Part E, 45, 2009, pp.878-892.

20. Sahin, C., Kuvvetli, Y., "Differential evolution based metaheuristic algorithm for dynamic continuous berth allocation problem". Applied Mathematical Modelling, 40, 2016, pp.1067910688 .

21. Kim, K. H., Moon, K. C., "Berth scheduling by simulated annealing". Transportation Research Part B Methodological, 37(6), 2003, pp.541-560.
22 Liu, C., Zheng, L., Zhang. C., "Behavior perception-based disruption models for berth allocation and quay crane assignment problems". Computer \& Industrial Engineering, 97, 2016, pp.258275.

23. Golias, M., Portal, I., Konur, D., "Robust berth scheduling at marine container terminals via hierarchical optimization". Computers \& Operations Research, 41, 2014, pp.412-422.

24. Lee, Y., Chen, C. Y., "An optimization heuristic for the berth scheduling pro". European Journal of Operational Research, 196(2), 2009, pp.500-508.

25. Lai, K. K., Shih, K., "A study of container berth allocation". Journal of Advanced Transportation, 26(1), 1992, pp.45-60..

26. Imai, A., Etsuko N., Stratos, P., "Berth allocation with service priority", Transportation Research Part B Methodological, 37(5), 2003, pp.437-457.

27. Imai, A., Sun. X., Nishimura, E., "Berth allocation in a container port: using a continuous location space approach". Transportation Research Part B Methodological, 39(3), 2005, pp.199-221.

28. Fan L., Wang X., Yin J., Joint Scheduling of Berth-Quay Crane Based on Fair Principle. Industrial Engineering and Management, 2(22), 2017, pp.60-68. 